\title{
VIRTUAL BETTI NUMBERS OF MAPPING TORI OF 3-MANIFOLDS
}

\author{
CHRISTOFOROS NEOFYTIDIS
}

\begin{abstract}
Given a reducible 3-manifold $M$ with an aspherical summand in its prime decomposition and a homeomorphism $f: M \rightarrow M$, we construct a map of degree one from a finite cover of $M \rtimes_{f} S^{1}$ to a mapping torus of a certain aspherical 3-manifold. We deduce that $M \rtimes_{f} S^{1}$ has virtually infinite first Betti number, except when all aspherical summands of $M$ are virtual $T^{2}$-bundles. This verifies all cases of a conjecture of T.-J. $\mathrm{Li}$ and $\mathrm{Y}$. Ni, that any mapping torus of a reducible 3-manifold $M$ not covered by $S^{2} \times S^{1}$ has virtually infinite first Betti number, except when $M$ is virtually $\left(\#_{n} T^{2} \rtimes S^{1}\right) \#\left(\#_{m} S^{2} \times S^{1}\right)$. Li-Ni's conjecture was recently confirmed by Ni with a group theoretic result, namely, by showing that there exists a $\pi_{1}$-surjection from a finite cover of any mapping torus of a reducible 3-manifold to a certain mapping torus of $\#_{m} S^{2} \times S^{1}$ and using the fact that free-by-cyclic groups are large when the free group is generated by more than one element.
\end{abstract}

\section{INTRODUCTION}

The virtual first Betti number of a manifold $M$ is defined to be

$$
v b_{1}(M)=\sup \left\{b_{1}(\bar{M}) \mid \bar{M} \text { is a finite cover of } M\right\}
$$

(where $b_{1}$ denotes the first Betti number) and takes values in $\mathbb{N}_{0} \cup\{\infty\}$. This notion arises naturally in geometric topology and it is often difficult to compute. A recent prominent example is given by the resolution of the Virtual Haken Conjecture [1] which implies that $v b_{1}=\infty$ for hyperbolic 3 -manifolds, and therefore completes the picture for the values of $v b_{1}$ in dimension three. $\mathrm{Li}$ and Ni [10] used this picture to compute $v b_{1}$ for mapping tori of prime 3-manifolds:

Theorem 1.1. ([10, Theorem 1.2]). Let $X=M \rtimes_{f} S^{1}$ be a mapping torus of a closed prime 3-manifold $M$. Then $v b_{1}(X)$ is given as follows:

(1) If $M$ is a spherical manifold, then $v b_{1}(X)=1$;

(2) If $M$ is $S^{1} \times S^{2}$ or finitely covered by $T^{2} \rtimes S^{1}$, then $v b_{1}(X) \leq 4$;

(3) In all other cases, $v b_{1}(X)=\infty$.

When the fiber $M$ is reducible, then the monodromy $f$ of the mapping torus $M \rtimes_{f} S^{1}$ is in general more complicated than when $M$ is irreducible; see [11, 14, 12]. Li and Ni conjectured that almost always $v b_{1}\left(M \rtimes_{f} S^{1}\right)=\infty$ when $M$ is reducible:

Conjecture 1.2. ([10, Conjecture 5.1]). If $M$ is a closed oriented reducible 3-manifold, then $v b_{1}\left(M \rtimes_{f} S^{1}\right)=\infty$, unless $M$ is finitely covered by $S^{2} \times S^{1}$.

Date: February 19, 2020.

2010 Mathematics Subject Classification. 57M05, 57M10, 57M50, 55M25, 57N37.

Key words and phrases. Virtual Betti numbers, mapping tori of reducible 3-manifolds, degree one maps. 
As pointed out in [10, Lemma 5.4] (see Lemma 2.2), if $M$ is a closed reducible 3-manifold which is not covered by $S^{2} \times S^{1}$, then $M$ is finitely covered by a connected sum $M^{\prime} \#\left(S^{2} \times S^{1}\right) \#\left(S^{2} \times S^{1}\right)$, for some closed 3-manifold $M^{\prime}$. Since free-by-cyclic groups are large whenever the free group is generated by more than one element (cf. [15, 7, 5]), we deduce that

$$
v b_{1}\left(\left(\#_{m} S^{2} \times S^{1}\right) \rtimes_{f} S^{1}\right)=\infty \text { for } m \geq 2 .
$$

Thus, when $M$ contains an aspherical summand in its prime decomposition, and not only summands covered by mapping tori of $T^{2}$, Conjecture 1.2 follows by the following result:

Theorem 1.3. Let $M$ be a closed oriented reducible 3-manifold that contains at least one aspherical summand in its prime decomposition. For any mapping torus $M \rtimes_{f} S^{1}$, there is a finite cover $\bar{M}$ of $M$ containing an aspherical summand $M_{1}$ in its prime decomposition and a degree one map

$$
\bar{M} \rtimes_{f^{k}} S^{1} \longrightarrow M_{1} \rtimes_{h} S^{1}
$$

for some $k \geq 1$ and some homeomorphism $h: M_{1} \longrightarrow M_{1}$.

Recently, Ni [13] verified Conjecture 1.2 by showing that there exists a surjection from the fundamental group of a mapping torus of a finite cover of $M$ to the fundamental group of a mapping torus of a connected sum $\#_{m} S^{2} \times S^{1}, m \geq 2$, and making use of the fact that free-by-cyclic groups with at least three generators are large. Our result is in a sense both stronger and weaker than Ni's result. It is stronger, on the one hand, because it comes with a construction of a map of nonzero degree, instead of just a $\pi_{1}$-surjection as in [13]. Indeed, it is likely that there is even a degree one map from a mapping torus of a finite covering of $M$ to a mapping torus of a connected sum $\#_{m} S^{2} \times S^{1}$; see [13, p. 1592]. However, the map we construct here makes essential use of the asphericity of the summand $M_{1}$ and therefore our method cannot be extended to the case where no aspherical summand exists in the prime decomposition of $M$. On the other hand, the $\pi_{1}$-surjection obtained by Ni covers as well the case where the aspherical summands of $M$ are only virtual mapping tori of $T^{2}$. Therefore, it is natural to ask whether one can find a topological proof of Conjecture 1.2 for connected sums of type $\left(\#_{n} T^{2} \rtimes S^{1}\right) \#\left(\#_{m} S^{2} \times S^{1}\right)$. Also, it would be interesting to find a purely group theoretic proof that for every reducible 3 -manifold $M$ that is not finitely covered by $S^{2} \times S^{1}$, any $\pi_{1}(M)$-by-cyclic group is large.

Outline. In Section 2 we give some facts about finite coverings of mapping tori and in Section 3 we recall the description of self-homeomorphisms of closed reducible 3-manifolds. The main body of the proof of Theorem 1.3 is given in Sections 4 and 5. Finally, we discuss Conjecture 1.2 in Section 6 .

Acknowledgements. The author is supported by the Swiss NSF, under grant FNS200021_169685. Part of this work was done during author's visit at IHÉS in 2018. The author would like to thank IHÉS for providing a stimulating working environment, and especially Misha Gromov and Fanny Kassel for their hospitality. 


\section{PRELIMINARIES}

We begin our discussion by gathering some well-known facts needed for our proofs.

Let $M$ be a closed oriented reducible 3-manifold. By the Kneser-Milnor theorem [8], $M$ can be decomposed as a connected sum

$$
M=M_{1} \# \cdots \# M_{n} \#\left(\#{ }_{m} S^{2} \times S^{1}\right) \#\left(\#_{p=1}^{s} S^{3} / Q_{p}\right)
$$

where each $M_{i}$ is aspherical and $S^{3} / Q_{p}$ are spherical quotients with fundamental groups the finite groups $Q_{p}$.

The following lemmas give some precise descriptions of finite covers of $M$ and can be found in [9, pp. 23-24] and [10, Lemma 5.4] respectively:

Lemma 2.1. If $n=0$ and $M \neq \mathbb{R} P^{3} \# \mathbb{R} P^{3}$ or $S^{2} \times S^{1}$, then $M$ is finitely covered by $\#_{m^{\prime}} S^{2} \times S^{1}$, for some $m^{\prime} \geq 2$.

Proof. Let the projection

$$
\varphi: \pi_{1}(M) \longrightarrow Q_{1} \times \cdots \times Q_{s}
$$

of the free product $\pi_{1}(M)=F_{m} * Q_{1} * \cdots * Q_{s}$ to the direct product $\prod_{p=1}^{s} Q_{p}$. By the Kurosh subgroup theorem, the kernel of $\varphi$ is a free group, say $F_{m^{\prime}}$, where $m^{\prime} \geq 2$. Since moreover $\operatorname{ker}(\varphi)$ has finite index in $\pi_{1}(M)$, Grushko's theorem implies that $M$ is finitely covered by the connected sum $\#_{m^{\prime}} S^{2} \times S^{1}$.

Lemma 2.2. If $n \geq 1$, then $M$ is finitely covered by $M^{\prime} \#\left(\#_{m^{\prime}} S^{2} \times S^{1}\right)$, where $M^{\prime}$ is a connected sum of aspherical 3-manifolds and $m^{\prime} \geq 2$.

Proof. Let $M=M_{1} \# M_{2}$, where $M_{1}$ is aspherical and $M_{2} \neq S^{3}$ (not necessarily prime). Since $\pi_{1}\left(M_{1}\right)$ is residually finite, there is a $d$-fold cover $\overline{M_{1}}$ of $M_{1}$ for some $d \geq 3$, and so $M$ is $d$-fold covered by $\bar{M}=\overline{M_{1}} \#\left(\#{ }_{d} M_{2}\right)$. Now, since $\pi_{1}\left(M_{2}\right)$ is residually finite, there is a finite group $G$ together with a surjection

$$
\psi: \pi_{1}(\bar{M}) \longrightarrow G
$$

which maps $\pi_{1}\left(\overline{M_{1}}\right)$ to the trivial element and each $\pi_{1}\left(M_{2}\right)$ surjectively to $G$. Then, since $G$ is finite, it is easy to see that the cover of $\bar{M}$ corresponding to $\operatorname{ker}(\psi)$ contains at least $m^{\prime}:=d-1 \geq 2$ connected summands $S^{2} \times S^{1}$.

Finally, we quote two general facts about coverings of mapping tori whose proof is easy and left to the reader (see also [10, Section 2]).

Lemma 2.3. Let $f: M \longrightarrow M$ be a self-homeomorphism of a closed oriented manifold (of any dimension).

(a) $M \rtimes_{f} S^{1}$ is finitely covered by $M \rtimes_{f^{k}} S^{1}$ for every $k \geq 1$.

(b) If $\bar{M}$ is a finite cover of $M$, then $M \rtimes_{f} S^{1}$ is finitely covered by $\bar{M} \rtimes_{f^{k}} S^{1}$ for some $k$. 


\section{SELF-HOMEOMORPHISMS OF REDUCIBLE 3-MANIFOLDS}

In this section, we recall the isotopy types of orientation-preserving homeomorphisms of 3manifolds. For the most part we follow the description given in McCullough's survey paper [11], however we adapt some parts of his description in order to simplify our next arguments.

Suppose $M$ is a closed oriented reducible 3-manifold. By the discussion in Section 2, we may assume that $M$ does not contain any spherical quotients in its prime decomposition. Consider the following construction of $M$ : Let $W$ be a punctured 3-cell obtained by removing $n+m$ open 3-balls from a 3-sphere, and let

$$
S_{1}, S_{2}, \ldots, S_{n}, S_{n+1}, S_{n+2}, \ldots, S_{n+m}
$$

be its boundary components. For each of the $S_{i}, i=1, \ldots, n$, remove the interior of a 3-ball $D_{i}^{3}$ from $M_{i}$, and attach $M_{i}^{\prime}=M_{i}-\operatorname{int}\left(D_{i}^{3}\right)$ to $S_{i}$ along $\partial D_{i}^{3}$. Similarly, for each of the $S_{j}$, $j=n+1, \ldots, n+m$, remove the interior of a 3 -ball $D_{j}^{3}=D_{j}^{2} \times I_{j}$ from $S^{2} \times S^{1}$, and attach $\left(S^{2} \times S^{1}\right)_{j}^{\prime}=\left(S^{2} \times S^{1}\right)-\operatorname{int}\left(D_{j}^{2} \times I_{j}\right)$ to $S_{j}$ along $\partial D_{j}^{3}$.

Using the above construction, we now describe three types of homeomorphisms of $M$. We remark that two orientation-preserving homeomorphisms of $W$ are isotopic if and only if they induce the same permutation on the boundary components of $W$.

1. Homeomorphisms preserving summands. These are the homeomorphisms of $M$ which restrict to the identity on $W$. Note that this class of homeomorphisms includes the so-called "spins" of $S^{2} \times S^{1}$ as given following McCullough's construction of $M$; compare [11, Remark, p. 69].

2. Interchanges of homeomorphic summands. If $M_{i}$ and $M_{j}$ are two orientation-preserving homeomorphic summands, then a homeomorphism of $M$ can be constructed by fixing the rest of the summands, leaving $W$ invariant, and interchanging $M_{i}^{\prime}$ and $M_{j}^{\prime}$.

Similarly, we can interchange any two $S^{2} \times S^{1}$ summands, leaving $W$ invariant.

3. Slide homeomorphisms. For $i=1, \ldots, n$, let $\widehat{M}_{i}$ be obtained from $M$ by replacing $M_{i}^{\prime}$ with a 3-ball $B_{i}$. Let $\alpha$ be an arc in $\widehat{M}_{i}$ which meets $B_{i}$ only in its endpoints and $J_{t}$ an isotopy of $\widehat{M}_{i}$ that moves $B_{i}$ around $\alpha$, with $J_{0}=i d_{\widehat{M_{i}}}$ and $\left.J_{1}\right|_{B_{i}}=i d_{B_{i}}$. The homeomorphism

$$
s: M \longrightarrow M
$$

defined by

$$
\left.s\right|_{M-M_{i}^{\prime}}=\left.J_{1}\right|_{\widehat{M}_{i}-B_{i}} \text { and }\left.s\right|_{M_{i}^{\prime}}=\left.i d\right|_{M_{i}^{\prime}} .
$$

is called slide homeomorphism of $M$ that slides $M_{i}$ around $\alpha$. Starting with a different isotopy $J_{t}$, then $s$ changes by an isotopy and perhaps by a rotation about the boundary component $S_{i}$. Therefore each $\alpha$ might determine two isotopy classes of a slide homeomorphism. Note that if $T$ is the frontier of a regular neighborhood of $M_{i}^{\prime} \cup \alpha$ in $M$, then $T$ is a compressible torus and $s$ is isotopic to a certain Dehn twist about $T$.

Similarly, one can slide an $S^{2} \times S^{1}$ summand around an arc in $M-\left(S^{2} \times S^{1}\right)_{j}^{\prime}$. 
We remark that if $\alpha_{1}$ and $\alpha_{2}$ are two arcs meeting $B_{i}$ only in their endpoints, and $\alpha$ represents their product, then a slide of $M_{i}$ around $\alpha$ is isotopic to a composite of slides around $\alpha_{1}$ and $\alpha_{2}$. Similarly for sliding $S^{2} \times S^{1}$.

With the above description, we have the following classification result of self-homeomorphisms of closed reducible 3-manifolds. This result was first announced in [6] and an elegant proof was given by McCullough [11, pp. 70-71], based on an argument of Scharlemann [4, Appendix A].

Theorem 3.1. ([11, p. 69]). If $M$ is a closed oriented reducible 3-manifold, then any orientationpreserving homeomorphism $f: M \longrightarrow M$ is isotopic to a composition of the following three types of homeomorphisms:

(1) homeomorphisms preserving summands;

(2) interchanges of homeomorphic summands;

(3) slide homeomorphisms.

In fact, the proof of Theorem 3.1 presented in [11, pp. 70-71], together with our adaptions on the construction of $M$, implies that

$$
f=g_{3} g_{2} g_{1}
$$

where $g_{3}$ is a finite composition of homeomorphisms of type 3 (slide homeomorphisms) and isotopies of $M$, and $g_{1}, g_{2}$ are compositions of finitely many homeomorphisms of type 1 and 2 respectively.

\section{Commutativity in HOMOTOPY}

Next, we show that there are self-homeomorphisms of an aspherical summand of a reducible 3-manifold whose "conjugation" by the pinch map in homotopy gives the three types of homeomorphisms described in the previous section.

According to the proof of Lemma 2.2, we may assume, after possibly passing to a finite cover, that $M$ contains an aspherical summand $M_{1}$ in its prime decomposition so that the selfhomeomorphism $f: M \longrightarrow M$ does not contain a component of $g_{2}$ that interchanges $M_{1}$ with another summand.

Clearly $M_{1}$ can be considered as being obtained by replacing each $M_{i}^{\prime}(i \geq 2)$ and $\left(S^{2} \times S^{1}\right)_{j}^{\prime}$ with a 3-ball $B_{i}$ and $B_{j}$ respectively. Then we can construct a pinch map

$$
p: M_{1} \# \cdots \# M_{n} \#\left(\#{ }_{m} S^{2} \times S^{1}\right) \longrightarrow M_{1}
$$

by mapping each $M_{i}^{\prime}(i \geq 2)$ to $B_{i}$, each $\left(S^{2} \times S^{1}\right)_{j}^{\prime}$ to $B_{j}$ and the rest of the part identically to itself.

We will show the following whose line of proof follows that of [13, Lemmas 3.6 and 3.7] adapted to our situation: 
Lemma 4.1. For each component $g_{i}$ of $f$, there is a self-homeomorphism $h_{i}$ of the aspherical summand $M_{1}$ such that the following diagram commutes in homotopy, i.e. $\left(p \circ g_{i}\right)_{*}=\left(h_{i} \circ p\right)_{*}$.

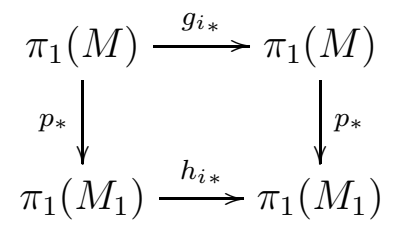

Proof. We will examine each of the (components of) $g_{i}$ separately.

Homeomorphisms preserving summands. Suppose first, that $g_{1}$ is a self-homeomorphism of summands $M_{i}$ or of $S^{2} \times S^{1}$. Define

$$
h_{1}: M_{1} \longrightarrow M_{1}
$$

by

$$
\left.h_{1}\right|_{M_{1}^{\prime}}=\left.g_{1}\right|_{M_{1}^{\prime}} \text { and }\left.h_{1}\right|_{M_{1}-M_{1}^{\prime}}=\left.i d\right|_{W \cup\left(\cup_{i=2}^{n} B_{i}\right) \cup\left(\cup_{j=1}^{m} B_{n+j}\right)} .
$$

If $\gamma$ is a loop in $M_{1}^{\prime}$, then $g_{1}(\gamma)$ is a loop in $M_{1}^{\prime}$ as well, and so

$$
p \circ g_{1}(\gamma)=g_{1}(\gamma)=g_{1} \circ p(\gamma)=h_{1} \circ p(\gamma) .
$$

If $\gamma \notin M_{1}^{\prime}$, then $g_{1}(\gamma) \notin M_{1}^{\prime}$, and since $W \cup\left(\cup_{i=2}^{n} B_{i}\right) \cup\left(\cup_{j=1}^{m} B_{n+j}\right)$ is simply connected, we deduce that $g_{1}(\gamma)$ and $p(\gamma)$ are homotopically trivial. Thus again $p_{*} \circ g_{1 *}=h_{1 *} \circ p_{*}$.

Interchanges of homeomorphic summands. Now, let $g_{2}$ be a homeomorphism that interchanges two aspherical summands $M_{i}$ and $M_{j}$ or two copies of $S^{2} \times S^{1}$. By our assumption on $M_{1}$, we know that $i, j \neq 1$. Set

$$
h_{2}: M_{1} \longrightarrow M_{1}, h_{2}:=i d
$$

If $\gamma$ is a loop in $M_{1}^{\prime}$, then $g_{2}(\gamma)=\gamma$, and so

$$
p \circ g_{2}(\gamma)=p(\gamma)=\gamma=h_{2}(\gamma)=h_{2} \circ p(\gamma) .
$$

If $\gamma \notin M_{1}^{\prime}$, then $g_{2}(\gamma) \notin M_{1}^{\prime}$, and so, as in the previous case, $p_{*} \circ g_{2 *}=h_{2 *} \circ p_{*}$, because $W \cup\left(\cup_{i=2}^{n} B_{i}\right) \cup\left(\cup_{j=1}^{m} B_{n+j}\right)$ is simply connected.

Slide homeomorphisms. Finally, let $g_{3}$ be a slide homeomorphism.

Suppose first that $g_{3}$ slides $M_{1}$ around an arc $\alpha$ in $M-M_{1}^{\prime}$ such that $\alpha \cap\left(M_{i}^{\prime}\right)$ and $\alpha \cap\left(S^{2} \times S^{1}\right)_{j}^{\prime}$ is a single arc for any $i \geq 2$ and any $j$. By McCullough's description, there is a Dehn twist $s_{\alpha}$ about the frontier $T$ of a regular neighbourhood of $M_{1}^{\prime} \cup \alpha$ which is isotopic to $g_{3}$. An arc $\beta$ in $W \cup\left(\cup_{i=2}^{n} B_{i}\right) \cup\left(\cup_{j=1}^{m} B_{n+j}\right)$ is given by letting $\left.\beta\right|_{W}$ be the same as $\left.\alpha\right|_{W}$ and $\left.\beta\right|_{\left(\cup_{i=2}^{n} B_{i}\right) \cup\left(\cup_{j=1}^{m} B_{n+j}\right)}$ be the trivial arc. Then we can define a Dehn twist $s_{\beta}$ about the frontier $T^{\prime}$ of a regular neighborhood of $M_{1}^{\prime} \cup \beta$ (corresponding to $g_{3}$ ). This defines our new homeomorphism $h_{3}: M_{1} \longrightarrow M_{1}$. If $\gamma$ is a loop in $M_{1}^{\prime}$, then clearly

$$
p \circ g_{3}(\gamma)=p(\gamma)=\gamma=h_{3}(\gamma)=h_{3} \circ p(\gamma) .
$$


If $\gamma \notin M_{1}^{\prime}$, then, after homotoping $\gamma$ if necessary, we can assume that $\gamma \cap T=\emptyset$ and $p(\gamma) \cap T^{\prime}=\emptyset$. We then have $g_{3}(\gamma)=s_{\alpha}(\gamma)=\gamma$ and so $p \circ g_{3}(\gamma)=p(\gamma)$ is homotopically trivial, because $W \cup\left(\cup_{i=2}^{n} B_{i}\right) \cup\left(\cup_{j=1}^{m} B_{n+j}\right)$ is simply connected. Thus $p_{*} \circ g_{3 *}=h_{3 *} \circ p_{*}$ as required.

Next, assume that $g_{3}$ slides some $M_{i}, i \neq 1$, around an arc in $M-M_{i}^{\prime}$ (similarly for sliding a copy of $S^{2} \times S^{1}$ ). We can assume that $\alpha \cap M_{1}^{\prime}$ is not trivial, otherwise the proof is identical to the above argument. Now, we have an $\operatorname{arc} \beta$ which is given by $\alpha$ in $M_{1}^{\prime} \cup W$ and it is trivial in $\left(\cup_{i=2}^{n} B_{i}\right) \cup\left(\cup_{j=1}^{m} B_{n+j}\right)$, and Dehn twists $s_{\alpha}$ and $s_{\beta}$ about tori $T$ and $T^{\prime}$, given similarly as above. In this way, we define our homeomorphism $h_{3}: M_{1} \longrightarrow M_{1}$. For loops not in $M_{1}^{\prime}$ the situation is as before, because $p(\gamma)$ is homotopically trivial. For a loop $\gamma$ in $M_{1}^{\prime}$, we can assume again that, after homotoping $\gamma$, we have $\gamma \cap T=\emptyset$ and $\gamma \cap T^{\prime}=\emptyset$. Then

$$
p \circ g_{3}(\gamma)=p \circ s_{\alpha}(\gamma)=p(\gamma)=\gamma=s_{\beta}(\gamma)=h_{3} \circ p(\gamma)
$$

This finishes the proof of the lemma.

\section{Finishing The PROOF OF THEOREM 1.3}

Now we will construct a map of degree one from $M \rtimes_{f} S^{1}$ to $M_{1} \rtimes_{h} S^{1}$, for some homeomorphism $h: M_{1} \longrightarrow M_{1}$.

As above, we can assume by Lemmas 2.1 and 2.2 that $M=M_{1} \# \cdots \# M_{n} \#\left(\#{ }_{m} S^{2} \times S^{1}\right)$, where $M_{i}$ are aspherical and $n \geq 1$. Moreover, we assume that $M_{1}$ is not interchanged under $f$ with another summand $M_{i}$ (by the proof of Lemma 2.2).

Consider the classifying space $B \pi_{1}(M)=M_{1} \vee \cdots \vee M_{n} \vee\left(\vee_{m} S^{1}\right)$ and the homotopically unique map

$$
B\left(f_{*}\right): M_{1} \vee \cdots \vee M_{n} \vee\left(\vee_{m} S^{1}\right) \longrightarrow M_{1} \vee \cdots \vee M_{n} \vee\left(\vee_{m} S^{1}\right),
$$

where $f_{*}: \pi_{1}(M) \longrightarrow \pi_{1}(M)$ is the isomorphism induced by $f$. Let also the map

$$
B\left(p_{*}\right): M_{1} \vee \cdots \vee M_{n} \vee\left(\vee_{m} S^{1}\right) \longrightarrow M_{1},
$$

induced by the pinch map $p$. (Again, $p_{*}: \pi_{1}\left(M_{1}\right) * \cdots * \pi_{1}\left(M_{n}\right) * F_{m} \longrightarrow \pi_{1}\left(M_{1}\right)$ denotes the induced homomorphism.)

By Theorem 3.1 (and the comments after that), we know that $f=g_{3} g_{2} g_{1}$, where $g_{3}$ is a finite composition of homeomorphisms of type 3 and isotopies of $M$, and each of $g_{1}, g_{2}$ is a composition of finitely many homeomorphisms of type 1 and 2 respectively, as given in Section 3 .

By Lemma 4.1, there is a homeomorphism $h: M_{1} \longrightarrow M_{1}$ such that

$$
p_{*} \circ f_{*}=h_{*} \circ p_{*} \text {. }
$$

For set $h=h_{3} h_{2} h_{1}$, where each (component of) $h_{i}$ is given by Lemma4.1. Then applying successively Lemma 4.1 on each $h_{i}$ we deduce that (5.1) indeed holds. Therefore, there is a well-defined surjective homomorphism

$$
\bar{p}_{*}: \pi_{1}\left(M \rtimes_{f} S^{1}\right) \longrightarrow \pi_{1}\left(M_{1} \rtimes_{h} S^{1}\right)
$$


which maps each element $x$ of $\pi(M)$ to $p_{*}(x) \in \pi_{1}\left(M_{1}\right)$ and the generator of the infinite cyclic group acting (through $f_{*}$ ) on $\pi_{1}(M)$ to the generator of the infinite cyclic group acting (through $\left.h_{*}\right)$ on $\pi_{1}\left(M_{1}\right)$.

Remark 5.1. If we replace $M_{1}$ by the connected sum $\#_{m} S^{2} \times S^{1}$ and adapt accordingly Lemma 4.1 to the situation of [13, Lemmas 3.6 and 3.7], then we will obtain a surjection

$$
\pi_{1}\left(M \rtimes_{f} S^{1}\right) \longrightarrow F_{m} \rtimes_{h_{*}} \mathbb{Z} .
$$

This surjection does not yield a map of non-zero degree, because the classifying space of $F_{m}$ is one dimensional.

The homomorphism $\bar{p}_{*}$ gives rise to a well-defined map

$$
B\left(\bar{p}_{*}\right): B \pi_{1}\left(M \rtimes_{f} S^{1}\right) \longrightarrow B \pi_{1}\left(M_{1} \rtimes_{h} S^{1}\right) .
$$

Since $M_{1}$ is aspherical, the homotopy long exact sequence for $\pi_{1}\left(M_{1} \rtimes_{h} S^{1}\right)$ implies that $M_{1} \rtimes_{h} S^{1}$ is aspherical. Furthermore,

$$
\pi_{1}\left(M \rtimes_{f} S^{1}\right)=\left\langle\pi_{1}(M), t \mid t x t^{-1}=f_{*}(x), x \in \pi_{1}(M)\right\rangle=\pi_{1}\left(B \pi_{1}(M) \rtimes_{B\left(f_{*}\right)} S^{1}\right) .
$$

Again, by the asphericity of $B \pi_{1}(M)$, we deduce that $B \pi_{1}(M) \rtimes_{B\left(f_{*}\right)} S^{1}$ is aspherical. Thus $B\left(\bar{p}_{*}\right)$ is (homotopic to) a map

$$
\begin{aligned}
B \pi_{1}(M) \rtimes_{B\left(f_{*}\right)} S^{1} & \longrightarrow M_{1} \rtimes_{h} S^{1} \\
{[(a, t)] } & \mapsto\left[\left(B\left(p_{*}\right)(a), t\right)\right],
\end{aligned}
$$

which we still denote by $B\left(\bar{p}_{*}\right)$.

Define now a map

$$
F: M \rtimes_{f} S^{1} \longrightarrow M_{1} \rtimes_{h} S^{1}
$$

by

$$
F:=B\left(\bar{p}_{*}\right) \circ \psi_{M \rtimes_{f} S^{1}},
$$

where $\psi_{M \rtimes_{f} S^{1}}: M \rtimes_{f} S^{1} \longrightarrow B \pi_{1}(M) \rtimes_{B\left(f_{*}\right)} S^{1}$ is the classifying map for $M \rtimes_{f} S^{1}$ (recall that $\left.\pi_{1}\left(M \rtimes_{f} S^{1}\right)=\pi_{1}\left(B \pi_{1}(M) \rtimes_{B\left(f_{*}\right)} S^{1}\right)\right)$. Let also $\psi_{M}: M \longrightarrow B \pi_{1}(M)$ denote the classifying map for $M$. Then the following diagram

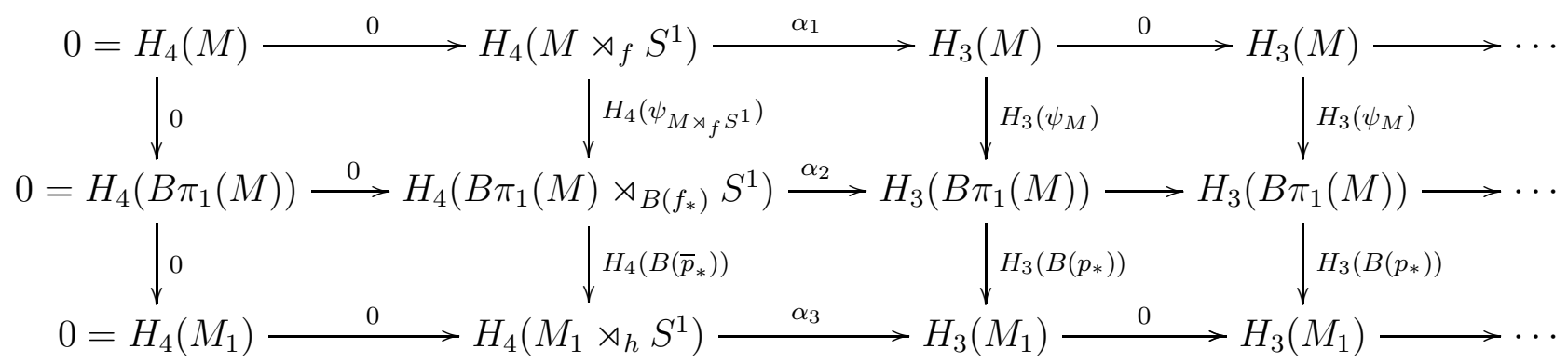


implies

$$
\begin{aligned}
\alpha_{3} \circ H_{4}\left(B\left(\bar{p}_{*}\right)\right) \circ H_{4}\left(\psi_{M \rtimes_{f} S^{1}}\right)\left(\left[M \rtimes_{f} S^{1}\right]\right) & =H_{3}\left(B\left(p_{*}\right)\right) \circ \alpha_{2} \circ H_{4}\left(\psi_{M \rtimes_{f} S^{1}}\right)\left(\left[M \rtimes_{f} S^{1}\right]\right) \\
& =H_{3}\left(B\left(p_{*}\right)\right) \circ H_{3}\left(\psi_{M}\right) \circ \alpha_{1}\left(\left[M \rtimes_{f} S^{1}\right]\right) \\
& =H_{3}\left(B\left(p_{*}\right)\right) \circ H_{3}\left(\psi_{M}\right)([M]) \\
& =H_{3}\left(B\left(p_{*}\right)\right)\left(\left[M_{1}\right], \ldots,\left[M_{n}\right]\right)=\left[M_{1}\right] .
\end{aligned}
$$

This means that

$$
H_{4}(F)\left(\left[M \rtimes_{f} S^{1}\right]\right)=\left[M_{1} \rtimes_{h} S^{1}\right]
$$

completing the proof of Theorem 1.3 .

\section{ViRTUAL FIRST BETTI NUMBERS}

In this section we discuss Conjecture 1.2

Recall that the finiteness of virtual first Betti numbers of mapping tori of prime 3-manifolds follows that of their fiber $M$, namely $v b_{1}(M)$ (and $v b_{1}\left(M \rtimes_{f} S^{1}\right)$ ) is finite if and only if $M$ is virtually $S^{3}, S^{2} \times S^{1}$ or a $T^{2}$-bundle. More precisely, if a 3-manifold $M$ is finitely covered by $S^{3}, S^{2} \times S^{1}$ or a $T^{2}$-bundle, then $v b_{1}(M) \leq 3$, and the corresponding mapping tori of $M$ satisfy $v b_{1}\left(M \rtimes_{f} S^{1}\right) \leq v b_{1}(M)+1 \leq 4$ for any homeomorphism $f: M \longrightarrow M$. It is therefore natural to examine Conjecture 1.2 according to whether a reducible 3-manifold contains a prime summand with virtually infinite first Betti number or not.

6.1. At least one prime summand with virtually infinite first Betti number. Suppose first that a reducible 3-manifold $M$ contains a summand in its prime decomposition with $v b_{1}=\infty$. This summand is necessarily aspherical. If $f: M \longrightarrow M$ is an orientation preserving homeomorphism, then Theorem 3.1 tells us that $f$ is isotopic to a composition $g_{3} g_{2} g_{1}$ where each $g_{i}$ is a finite composition of homeomorphisms of type $i=1,2,3$. By Lemmas 2.1, 2.2 and 2.3 (and their proofs), there is a finite a cover $\bar{M}$ of $M$ containing an aspherical summand $M_{1}$ in its prime decomposition which is not virtually a mapping torus of $T^{2}$ and is not interchanged by some $f^{k}$ (under a component of type 2) with any other summand of $\bar{M}$. Then Theorem 1.3 implies that there is a self-homeomorphism $h$ of $M_{1}$ and a degree one map

$$
\bar{M} \rtimes_{f^{k}} S^{1} \longrightarrow M_{1} \rtimes_{h} S^{1} .
$$

In particular, $b_{1}\left(\bar{M} \rtimes_{f^{k}} S^{1}\right) \geq b_{1}\left(M_{1} \rtimes_{h} S^{1}\right)$, and so Theorem 1.1 implies that

$$
v b_{1}\left(M \rtimes_{f} S^{1}\right)=\infty .
$$

This proves Conjecture 1.2 in all cases, except when $M$ is virtually $\left(\#_{n} T^{2} \rtimes S^{1}\right) \#\left(\#{ }_{m} S^{2} \times S^{1}\right)$ (containing at least two summands). 
6.2. Only summands with virtually finite first Betti numbers. Suppose, finally, that $M$ is (virtually) of the form $\left(\#_{n} T^{2} \rtimes S^{1}\right) \#\left(\#_{m} S^{2} \times S^{1}\right)$. In that case, Theorem 1.3 is not anymore applicable to deduce that $v b_{1}\left(M \rtimes_{f} S^{1}\right)=\infty$. On the one hand, if $n=0$, then $\#_{m} S^{2} \times S^{1}$ does not contain any aspherical summands. On the other hand, if $n \neq 0$, then Theorem 1.3 implies that there is a degree one map $\bar{M} \rtimes_{f^{k}} S^{1} \longrightarrow\left(T^{2} \rtimes S^{1}\right) \rtimes_{h} S^{1}$, which, however, does not suffice to conclude that $v b_{1}\left(M \rtimes_{f} S^{1}\right)=\infty$ because $v b_{1}\left(\left(T^{2} \rtimes S^{1}\right) \rtimes_{h} S^{1}\right) \leq 4$. It would be interesting to find topological arguments that cover those two cases as well.

Nevertheless, we can appeal to group theoretic results to deduce that $v b_{1}=\infty$ in the remaining two cases. First, one can deduce from known results that $\pi_{1}\left(\left(\#_{m} S^{2} \times S^{1}\right) \rtimes_{f} S^{1}\right)$ is large for $m \geq 2$ : By [2, 3], the free-by-cyclic group

$$
F_{m} \rtimes_{f_{*}} \mathbb{Z}=\pi_{1}\left(\left(\#_{m} S^{1} \times S^{1}\right) \rtimes_{f} S^{1}\right), m \geq 2,
$$

is word hyperbolic if and only if it does not contain as subgroup an isomorphic copy of $\mathbb{Z}^{2}$. In the case where $F_{m} \rtimes_{f_{*}} \mathbb{Z}$ is hyperbolic, then it is large by [1, 7, 15]. If now $\mathbb{Z}^{2} \subset F_{m} \rtimes_{f_{*}} \mathbb{Z}$, then $F_{m} \rtimes_{f_{*}} \mathbb{Z}$ is large by [5]. Thus in all cases we deduce that $v b_{1}\left(F_{m} \rtimes_{f_{*}} \mathbb{Z}\right)=\infty$ as required.

Therefore, using the largeness of $F_{m} \rtimes_{f_{*}} \mathbb{Z}$, we conclude that

$$
v b_{1}\left(\left(\#_{m} S^{2} \times S^{1}\right) \rtimes_{f} S^{1}\right)=\infty \text { for } m \geq 2 .
$$

Remark 6.1. By [2, 3], $F_{m} \rtimes_{f_{*}} \mathbb{Z}$ being word hyperbolic is equivalent to the automorphism $f_{*}$ being atoroidal, i.e. having no non-trivial periodic conjugacy classes. When $f_{*}$ is toroidal (i.e. it has some non-trivial periodic conjugacy class), Ni also showed that $v b_{1}\left(F_{m} \rtimes_{f_{*}} \mathbb{Z}\right)=\infty$; see [13, Lemma 2.4].

Finally, as Ni shows in all cases where aspherical summands exist, our remaining case of mapping tori of $\left(\#_{n} T^{2} \rtimes S^{1}\right) \#\left(\#{ }_{m} S^{2} \times S^{1}\right)$, where $n \geq 1$, can be treated as follows: Recall that we can always assume that $m \geq 2$ and using the $\pi_{1}$-surjection induced by the pinch map

$$
\pi_{1}\left(\left(\left(\#_{n} T^{2} \rtimes S^{1}\right) \#\left(\#_{m} S^{2} \times S^{1}\right)\right) \rtimes_{f} S^{1}\right) \longrightarrow \pi_{1}\left(\left(\#_{m} S^{2} \times S^{1}\right) \rtimes_{h} S^{1}\right),
$$

we deduce that

$$
v b_{1}\left(\left(\left(\#_{n} T^{2} \rtimes S^{1}\right) \#\left(\#_{m} S^{2} \times S^{1}\right)\right) \rtimes_{f} S^{1}\right)=\infty
$$

as required; see also Remark 5.1.

\section{REFERENCES}

[1] I. Agol, The virtual Haken conjecture, with an appendix by I. Agol, D. Groves, and J. Manning, Doc. Math., 18 (2013), 1045-1087.

[2] M. Bestvina and M. Feighn, A combination theorem for negatively curved groups, J. Differential Geom., 35 (1992), 85-101.

[3] P. Brinkmann, Hyperbolic automorphisms of free groups, Geom. Funct. Anal., 10 (2000), 1071-1089.

[4] F. Bonahon, Cobordism of automorphisms of surfaces, Ann. Sci. École Norm. Sup. 16 (1983), $237-270$.

[5] J. O. Button, Large groups of deficiency 1, Isr. J. Math. 167 (2008), 111-140.

[6] E. César de Sá and C. Rourke, The homotopy type of homeomorphisms of 3-manifolds, Bull. Amer. Math. Soc. (N.S.) 1, no. 1 (1979), 251-254. 
[7] M. Hagen and D. Wise, Cubulating hyperbolic free-by-cyclic groups: The general case, Geom. Funct. Anal., 25 (2015), 134-179.

[8] J. Hempel, 3-manifolds, Princeton University Press And University of Tokyo Press, 1976.

[9] D. Kotschick and C. Neofytidis, On three-manifolds dominated by circle bundles, Math. Z. 274 (2013), 21-32.

[10] T.-J. Li and Y. Ni, Virtual Betti numbers and virtual symplecticity of 4-dimensional mapping tori Math. Z., 277 (2014), 195-208.

[11] D. McCullough, Mappings of reducible 3-manifolds, Proceedings of the Semester in Geometric and Algebraic Topology, Warsaw, Banach Center (1986), 61-76.

[12] C. Neofytidis and S. Wang, Invariant incompressible surfaces in reducible 3-manifolds, Ergodic Theory Dynam. Systems 39 (2019), 3136-3143.

[13] Y. Ni, Virtual Betti numbers and virtual symplecticity of 4-dimensional mapping tori, II, Sci. China Math. 60 no. 9 (2017), 1591-1598.

[14] X. Zhao, On the Nielsen numbers of slide homeomorphisms on 3-manifolds. Topology Appl. 136, no. 1-3 (2004), $169-188$.

[15] D. Wise, From Riches to Raags: 3-Manifolds, Right-Angled Artin Groups, and Cubical Geometry, CBMS Regional Conference Series in Mathematics 117, American Mathematical Society, Providence, RI, 2012.

Department of Mathematics, Ohio State University, Columbus, OH 43210, USA

E-mail address: neofytidis.1@osu.edu 